\title{
Chemotherapy of metastatic hepatoid adenocarcinoma: Literature review and two case reports with cisplatin etoposide
}

\author{
VICTOR SIMMET $^{1}$, MARGOT NOBLECOURT $^{1}$, THIBAUT LIZÉE $^{2}$, \\ BENJAMIN MORVANT ${ }^{3}$, SYLVIE GIRAULT ${ }^{4}$, PATRICK SOULIÉ ${ }^{1}$ and OLIVIER CAPITAIN ${ }^{1}$ \\ Departments of ${ }^{1}$ Medical Oncology and ${ }^{2}$ Radiotherapy, Integrated Center of Oncology (ICO) Paul Papin, \\ Angers 49055; ${ }^{3}$ Department of Pathologic, Angers University Hospital, Angers 49100; ${ }^{4}$ Department of \\ Nuclear Medicine, Integrated Center of Oncology (ICO) Paul Papin, Angers 49055, France
}

Received January 2, 2017; Accepted June 29, 2017

DOI: $10.3892 / \mathrm{ol} .2017 .7263$

\begin{abstract}
Hepatoid adenocarcinoma (HAC) is a rare and aggressive cancer subtype with a poor prognosis under metastatic conditions. Currently, there is no specific chemotherapy treatment protocol for advanced stages of the disease. This review evaluates two cases of HAC of gastric cardia with synchronous liver metastasis, which were successfully treated by chemotherapy with cisplatin $\left(25 \mathrm{mg} / \mathrm{m}^{2}\right.$ each day) (day 1 to day 3) and etoposide (100 $\left.\mathrm{mg} / \mathrm{m}^{2}\right)$ (day 1 to day 3), every three weeks. A structured literary evaluation and reviewed pertinent articles are additionally presented to analyse the different approaches for the treatment of metastatic HAC (mHAC). The two described case reports demonstrated good partial responses to treatment and one of the two patients exhibited a good prognosis after a 9-year follow-up. A total of 20 case reports concerning the use of chemotherapy in mHAC were presented in the literature, 11 of which were regarding gastric HACs. The two aforementioned cases result in a total of 22 reports, 11 of which exhibited objective responses to chemotherapy, 8 patients demonstrated a partial response and 3 a complete response. The cisplatin-based regimen concerned $55 \%(12 / 22)$ patients and enabled $9(75 \%)$ to exhibit a partial or complete response. A total of three patients exhibited a good prognosis in the long-term follow-up, all of them treated with a cisplatin-based regimen. It was demonstrated that the usual digestive regimens were not efficient in the treatment of HAC. In the absence of prospective trials, it may be hypothesized that cisplatin-based chemotherapy may be the most efficient first-line treatment in mHAC, with a $75 \%$ patient response, in accordance with the literature and follow-up cases.
\end{abstract}

Correspondence to: Dr Victor Simmet, Department of Medical Oncology, Integrated Center of Oncology (ICO) Paul Papin, 15 Rue André Boquel, 49055 Angers, France

E-mail: victor.simmet@gmail.com

Key words: gastric cancer, hepatoid adenocarcinoma, $\alpha$-fetoprotein, chemotherapy, cisplatin, etoposide

\section{Introduction}

Hepatoid adenocarcinoma (HAC) represents approximately 0.2 to $0.8 \%$ of diagnosed gastric cancer (1). Ishikura et al defined HAC as extrahepatic tumor with hepatocyte differentiation and potentially increased $\alpha$-fetoprotein (AFP) secretion (2). HAC diagnosis is mainly histological and is characterized by large and polygonal hepatocyte-like cells with intense eosinophil cytoplasm and big central nucleus. HAC is mainly located in oesophagus and stomach, however other sites were also described (3). According to a Chinese analysis, which includes a total of 180 gastric HACs from 62 different cases reports, the 3 -year survival rate was only of $7.36 \%$ and the median survival time of 10 months (1). Another analysis including 31 gastric HAC patients described that: i) $87 \%$ of them exhibited an increased secretion of AFP serum level; ii) 55\% had a locoregional synchronous node extension; and iii) $25 \%$ had synchronous metastatic, especially hepatic. An overexpression of AFP was detected by immunohistochemistry (IHC) in $90.3 \%$ of cases (4). Conversely, Nagai et al showed that in $46 \%$ of HAC patients, any increase of AFP secretion was observed. Therefore, they proposed a novel description of HAC as an extrahepatic tumor with typical hepatocyte cells with or without AFP secretion (5). Importantly, HAC must not be confounded with classical gastric adenocarcinoma, which can be associated with AFP overexpression, and having a better prognosis. Indeed, gastric HAC prognosis is poorer than classical histological types ones $(4,6)$. Currently, the treatment of metastatic HAC (mHAC) remains not elucidated. Herein, we report two case-reports of mHAC patients. Moreover, through a pertinent review of the literature, we aim to analyse the different current therapeutic approaches for mHAC.

\section{Materials and methods}

We have performed a PRISMA-compliant systematic review of literature concerning the use of chemotherapy in mHAC (7). Inclusion criteria for articles published from January 2001 to October 2016 were: i) mHAC; ii) treated by chemotherapy; and iii) with an evaluation of the response. The following term was used in the Pubmed and Science Direct database exploration: 'metastatic hepatoid carcinoma', or 'hepatoid' 
with mesh term 'drug therapy'. We also performed an investigation in the Grey literature database and in all case reports published in open case reports journals, which are not indexed in Pubmed databases (Journal of Medical Case Report, BMJ Case Report, American Journal of Case Report, International Journal of Case Reports, Clinical Case Reports) with the following term, 'hepatoid'. The flow chart respect PRISMA criteria, but no registration was carried out prospectively, explaining the lack of PROSPERO number (7). We identified 104 related reports in Pubmed Database and 9 in open case reports journals (Fig. 1). Though, no related article was found in Grey Literature. Thirty articles were not pertinent and 13 were not available in English full text. Sixty-eight full text articles were assessed for eligibility. Among them, 8 were not related to $\mathrm{HAC}, 10$ exhibited no metastasis features and 24 were not associated with chemotherapy. The nature of chemotherapy was not described in 10 case reports from 5 articles. The response to chemotherapy was not related in 9 case reports from 3 articles. Finally, only 18 articles concerning 20 patients were included. The best overall response (BOR), corresponding to the addition of best metastatic response (BMR) and the best primitive lesion response (BPR) to chemotherapy was reported and assessed according to Response Evaluation Criteria In Solid Tumors (RECIST version 1.1). The decrease of serum AFP levels following the treatment, named 'biological response' (BioR), was also assessed.

First case report. Mr. B., a 64-year-old French man, suffering from gastric reflux with hiatal hernia treated by proton inhibitor, was admitted to the gastroenterology department in February 2006 for epigastric and right hypochondrium pains associated with an anorexia and lose of weight. During the physical examination, the patient had a rapidly worsening general state with i) a Performans Status estimated to 4; ii) an icterus; iii) a paraneoplasic fever; and iv) a painful massive hepatomegaly. The laboratory investigation showed a biological chronic inflammation with LDH 25 times upper limit of normal (ULN), ASAT 2 ULN, and icteric cholestasis with total bilirubin at $120 \mu \mathrm{mol} / 1$. Carcinoembryonic antigen (CEA) and CA 19-9 were normal, but serum AFP was elevated to $2,600 \mathrm{ng} / \mathrm{ml}$. An oesogastroduodenoscopy discovered an ulcerated cardial tumoral lesion. Ultrasound scanning and computed tomography (CT) scans found three lesions in the right liver. A histopathologic examination of the cardia biopsy showed an ulcered HAC of the cardia, with tubulopapillary contingents. It was composed of large cells, with large nucleus, eosinophilic cytoplasm, and few hyaline balls. The periodic acid-Schiff (PAS) coloration, as well as the IHC of Hep Par antibodies, was negative. The histological examination of the hepatic biopsy showed the same HAC with the same cellular features, but with more necrosis aspects. The IHC study revealed CK19 positive (+), CK7 negative (-), CD10-, Chromogranine-, and Anti-synaptophysine negative cells.

A systemic chemotherapy was delivered associating cisplatin $25 \mathrm{mg} / \mathrm{m}^{2}$ with etoposide $100 \mathrm{mg} / \mathrm{m}^{2}$ each day, day 1 to day 3 , every 3 weeks. After one cycle, an impressive clinical improvement was notified with a decrease of abdominal pains and an extinction of hepatomegaly, with a straight decrease of icteric cholestasis although the AFP level was enhanced to

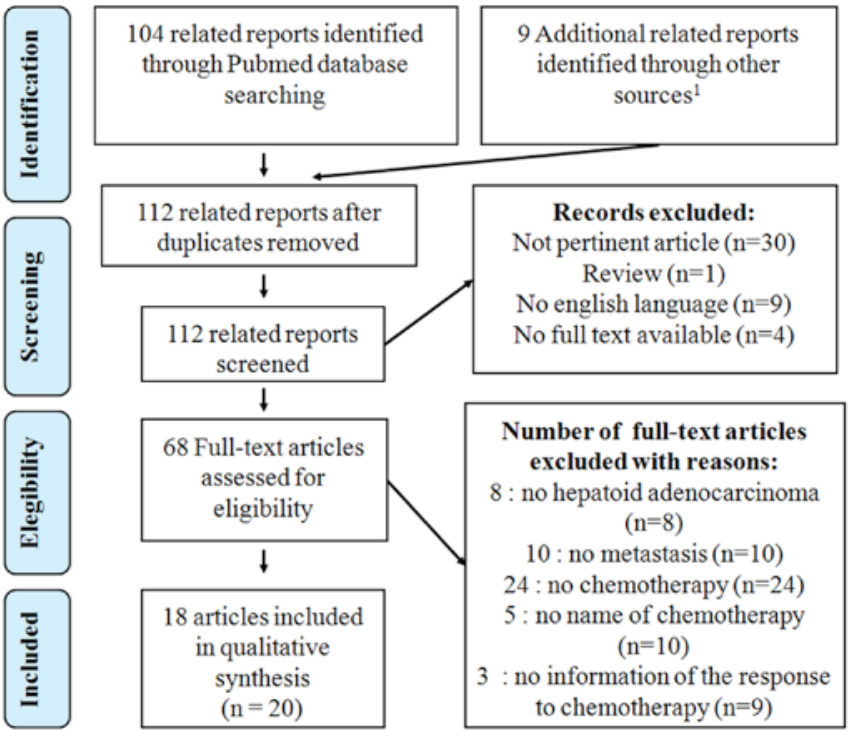

Figure 1. PRISMA-Compliant Flow chart of selection cases treated by chemotherapy in metastatic hepatoid adenocarcinoma: ${ }^{1}$ Grey literature and Open Case Report Journal not indexed in Pubmed. n, number of patients.

$6,900 \mathrm{ng} / \mathrm{ml}$. After 3 cycles, CT scans showed a good partial response on the liver metastasis and on the cardia lesion, confirmed after 6 cycles with a reduction of more than $80 \%$ of the size of liver metastasis. Moreover, the serum AFP levels were normalized. The Positron Emission Tomography (PET), realised two months after the end of chemotherapy, showed a complete metabolic gastric response and the persistence of two lesions in the segment VIII. The oesogastroduodenoscopy revealed an inflammatory cardia with complete histopathologic responses. After a one-year follow-up, a radical surgery associating gastrectomy and right hepatotectomy was decided by pluridisciplinary committee, and carried out. The patient was still alive, in complete remission at the last CT scan, more than 9 years after his diagnosis (Tables I and II; case 1).

Second case report. A 60 year-old French woman was admitted to the emergency ward in December 2014 for insomnia-related pains in her right hypochondrias, associated with anorexia, asthenia and a lose of weight of $3 \mathrm{~kg}$ in one month. Gastro-duodenal ulcer was notified in her digestive antecedents. The physical examination found a hepatomegaly and epigastric pains on the palpation. The laboratory investigation found ASAT $2 \mathrm{ULN}, \mathrm{LDH}$ to $7 \mathrm{ULN}$, and a non-icteric cholestasis. CT scans showed a tumor in the cardia extended to the low-oesophagus and greater curvature, numerous liver metastasis, up to $7 \mathrm{~cm}$, with hypervascularization and necrotic center in arterial phase. In Magnetic Resonance Imaging (MRI), cardia tumor and hepatic metastasis appeared hyper-intense in T2 and diffusion sequence, with peripheral arterial enhancement (ring-shape) without washout. PET revealed an intense hypermetabolism of the liver metastasis and primary lesion (Fig. 2). With particularly features in CT scans, tumoral markers have been analysed: $76,000 \mathrm{ng} / \mathrm{ml}$ for AFP, $176 \mathrm{IU} / \mathrm{ml}$ for CEA, and normal CA19-9. Oesogastroduodenoscopy showed an infiltrated ulceration of $4 \mathrm{~cm}$ on low-oesophagus and cardia on the third 


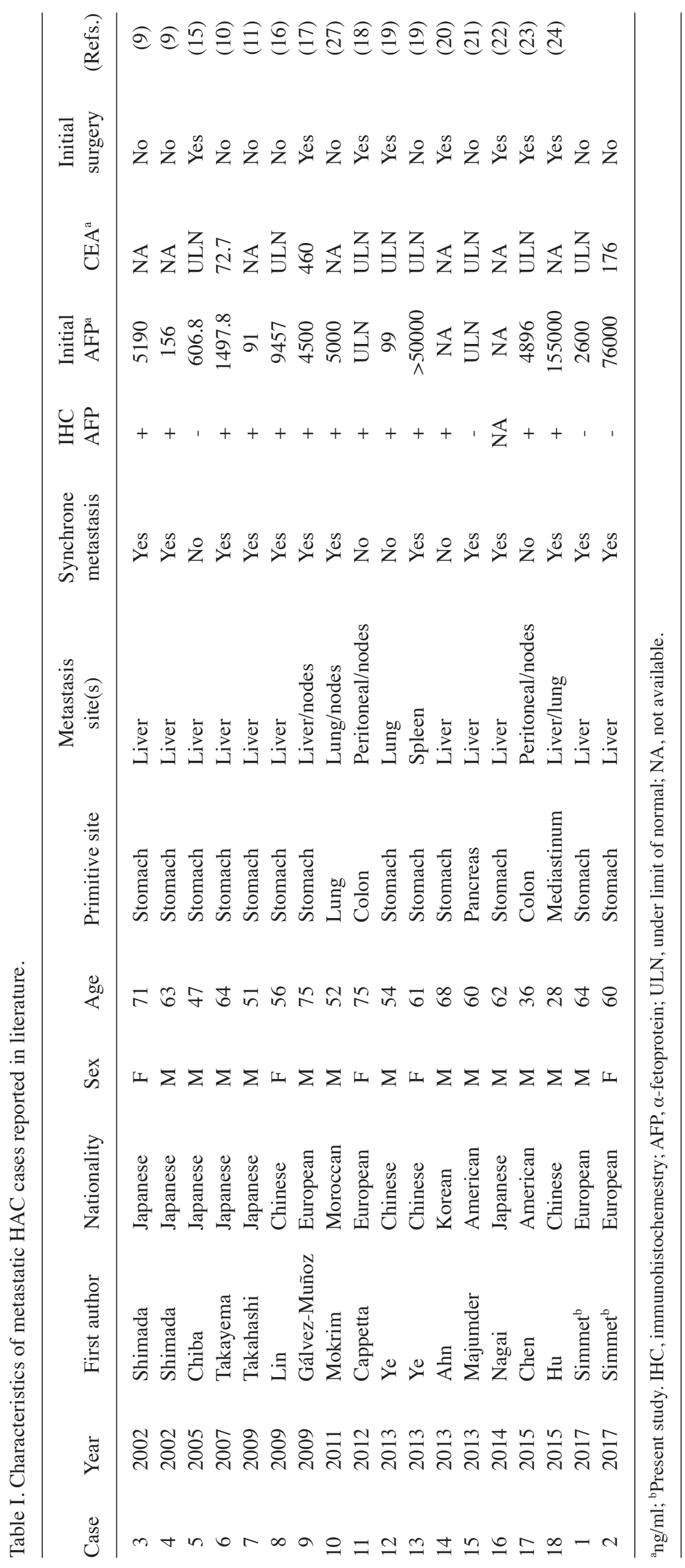




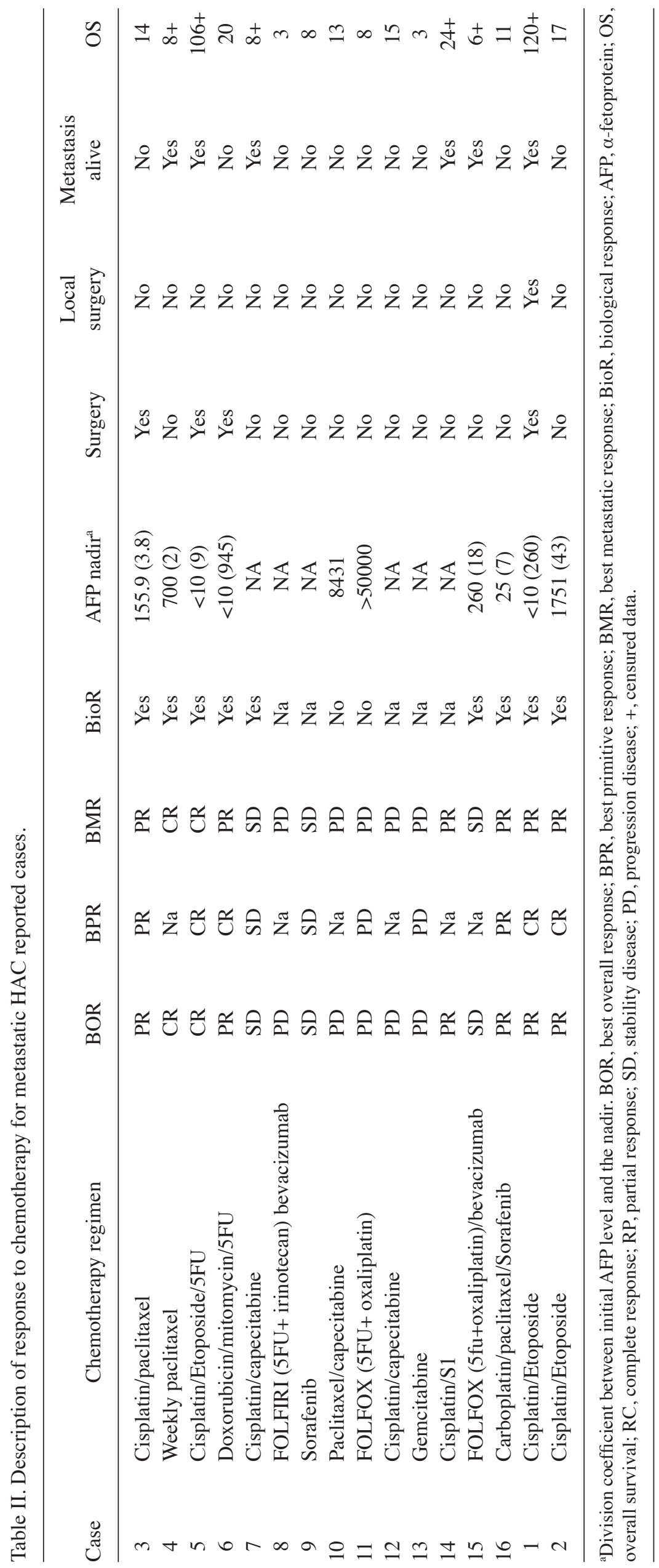




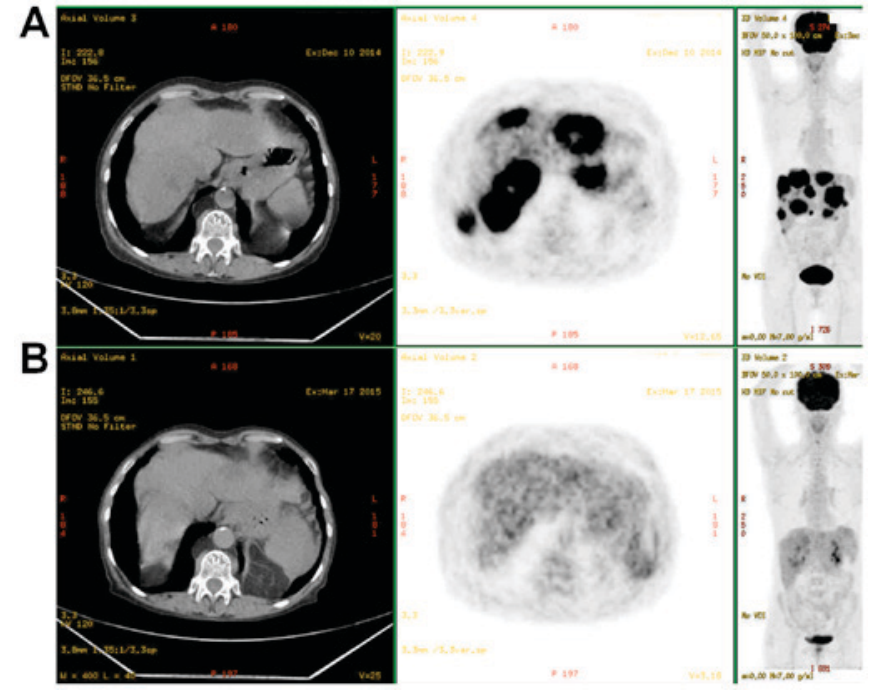

Figure 2. Second patient. Evaluation of the response to positron emission tomography (A) before and (B) after 3 cisplatin etoposide cycles.

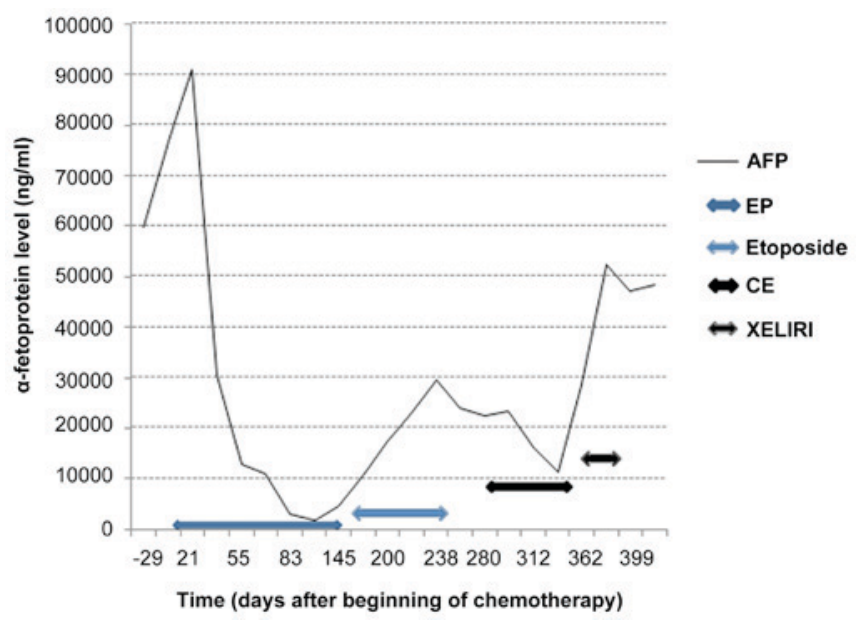

Figure 3. Second Patient. Evaluation of $\alpha$-fetoprotein level, before and after chemotherapy. AFP, $\alpha$-fetoprotein; EP, etoposide + cisplatin; CE, carboplatin + etoposide; XELIRI, irinotecan + capecitabin.

of the curvature. The histopathologic examination confirmed a HAC: round cells with big nuclei not much nucleolated, occasionally associated with a very dense chromatin, an eosinophile cytoplasm, but without intracytoplasmic hyaline globules as evaluated by PAS. As revealed by IHC studies, tumors were found KL1 positive (+), Glypican+, Glutamine Synthetase+, CK5-6 low, Hep Par antibodies low, CK20 negative (-), CK5-, AFP negative, Betacatenine-, Synaptophysin-, and P63 or Oestrogen receptors negative.

A systemic chemotherapy was delivered associating cisplatin $25 \mathrm{mg} / \mathrm{m}^{2}$ with etoposide $100 \mathrm{mg} / \mathrm{m}^{2}$ each day, day 1 to day 3 , every 3 weeks, with a rapid decrease of pain, after the first cycle, although an initial increase of tumoral markers was observed (Fig. 3). The first evaluation after 3 cycles by PET showed a metabolic complete response on the primary oesogastric lesion and metabolic partial response on the metastatic liver lesions (Fig. 2). After 6 cycles, persistence of complete response was confirmed on the primary lesion, but an increase in size and intensity of liver metastasis was observed. A maintenance therapy by oral etoposid was administrated, but stopped 3 months later due to marker's increase. Nowadays, PET scan confirmed a hepatic progression with appearance of hypermetabolism on the left lower paratracheal nodes, without any relapse in oesogastric junction. A second line treatment with carboplatin and etoposid was carried out leading to a novel decrease of AFP levels before observing any progression (Fig. 3). Finally, a third treatment by XELIRI (capecitabin and irinotecan) was administrated, without any efficiency. The patient died after 23 months-follow-ups (Tables I and II; case 2).

\section{Results}

Only one retrospective Korea cohort of 13 mHAC was found in our study with: i) one partial response reported with 5-fluoruracil (5-FU) associated with cisplatin; and ii) a second one with cisplatin-paclitaxel in second line (8). Nevertheless, the others patients received different chemotherapy regimens. Therefore, as the nature of the treatment for each patients remained unknown, this cohort was excluded for the final analysis. Moreover, we have reported 2 colon, 2 lung, 2 pancreas, 1 bladder, 1 peritoneal, 1 mediastinum mHACs and 11 gastric mHACs (Tables I and III, especially with sorafenib treatment). Including our two case reports, the median age of the diagnosis was 56 years. Patients $(73 \%)$ were male, 63\% (14/22) and 72\% (16/22) exhibited liver metastasis and synchrone metastasis, respectively. IHC AFP study was positive in $75 \%$ of cases $(15 / 20)$. When increased, the average AFP serum level was of $16,597 \mathrm{ng} / \mathrm{ml}$. An initial surgery was carried out in $41 \%$ of cases $(9 / 22)$. 11/22 BOR were observed with chemotherapy. Among them, 8/22 were partial responses and 3/22 complete ones. The complete response concerned 3 Japanese male patients with gastric HAC and liver metastasis. 55\% (12/22) of the patients received a cisplatin-based regimen, whose 9/12 had partial or complete responses. Among partial or complete response of all case reports, 9/11 (81\%) had a cisplatin-based chemotherapy. One another partial response was observed with a triple combined therapy: mitomycin-C, doxorubicin and 5-fluorouracil (Table II; case 8).

Three patients were still alive with a follow-up superior to one year; these 3 patients were under Cisplatin-based chemotherapy regimen (Table II; cases 1, 7 and 14). Finally, BioR was observed in 14 of 16 evaluable patients and AFP serum levels were normalized in 4 of 15 included patients of our review (Table II; cases 1, 4, 7 and 8).

In addition, 4 patients were treated by sorafenib in literature (Table III). One of them, presenting a lung mHAC, also received an additional lung-chemotherapy regimen (Table III; case 22) (12). Three other patients were treated in monotherapy, which permitted a greater stability of the disease (Table III; cases 19, 20 and 21).

\section{Discussion}

Based on our investigation, we assume to describe the first two case reports of mHAC treated by cisplatin-etoposid as first-line chemotherapy leading to major partial and complete responses considering the primitive lesion. Indeed, 
we report a complete biological response: after a nine-year follow-up, we consider the patient to be completely treated from mHAC (described as the first case report). The treatment efficiency seems to be mostly due to cisplatin as we could detect a recurrence on the liver metastasis under oral etoposid in the case 2 . As the maximal dose of administrated cisplatin had to be taken into account, this was preventing an additional cisplatin regimen in case 2 . As described in case 2 , other chemotherapy regimens appeared as inefficient as second and third lines treatment.

The review of literature is very limited and very few articles concerning the treatment of mHACs were reported. Lots of articles had to be excluded because no data regarding neither the kind of used chemotherapy nor its response were described. Therefore, these results had to be carefully interpreted and analysed. Indeed, as it is only an analysis of retrospective cases report, we cannot avoid some publication bias. In addition, the dose and schedules of chemotherapy administration were often not described in literature. Our results revealed a partial or complete response in 11 cases reports whom $81 \%$ had a Cisplatin-based regimen. Only one case, treated by cisplatin-etoposide-5-FU, can be identified as an efficient treatment with a long follow-up.

In our sense, cisplatin is probably the most interesting regimen to treat mHACs. These results were strongly correlated with ex vivo studies. Indeed, in a xenograft model of AFP-producing gastric cancer, mitomycin-C and cisplatin treatments might be effective to induce suppression of tumor growth, whereas 5-FU, doxorubicin, and epirubicin were shown as inefficient (13). In our review, two complete responses have been observed with a cisplatin-based regimen or mitomycin-C regimen. Other usual chemotherapies carried out in digestive cancer as irinotecan, oxaliplatin, gemcitabine or 5-FU, are appearing as inefficient in mHAC (Table II; cases 11, 13, 15 and 17).

Concerning targeted therapies, we do not possess full information. We can suppose that sorafenib which is the reference treatment in non-operable hepatocellular carcinoma could be a treatment option in HAC since they have similar histological features. An interesting progression free survival was reported in our review (Table III). In addition, an analysis of peritoneal mHAC in a 21-year-old man, showed a strong activation of the epidermal growth factor, and of the kinases ERK1 and AKT1 (14). Performing genome sequencing could be interesting in order to understand the molecular biology underlying mHAC (not described in the present time). In our cases, histopathological material was old or little biopsy materials, which is a strong limit to carry out a next sequencing generation.

Concerning non-gastric mHAC, we have stated only 2/7 partial responses in a lung and mediastinum primitive, and no objective responses in the two cases of colorectal HACs treated by a combined therapy composed of FOLFOX (oxaliplatin and 5-FU) plus bevacizumab, or FOLFIRI (irinotecan and 5-FU) plus bevacizumab (Table II; cases 11 and 17, respectively). No objective responses were related in a pancreatic HAC treated by gemcitabine. Consequently, traditional regimens seem to be not efficiency in mHAC. Cisplatin-based regimen seems to be the best option in non-gastric primitive HAC. Moreover radical surgery of primitive lesion or residual 
lesion could be ever considered and discussed again in case of good and durable response in mHACs as reported in the case 1 .

In conclusion, HAC represents a very rare and aggressive subtype of cancer defined as extrahepatic tumor with hepatocyte differentiation and potentially an increased AFP secretion. Currently, the treatment of mHAC remains not elucidated. We reported two cases of patients with mHAC successfully treated by chemotherapy with cisplatin and etoposide as first line leading to complete response. One of them is still alive after more than 9 years. In the absence of prospective trials in this rare cancer, we assume that cisplatin-based chemotherapy regimen could be the best first-line treatment in mHAC and considering as well our real-life experience as in view of literature, with $81 \%$ of response. Since the data of genome-wide analysis in cancers, it might be useful to provide some information with next generation sequencing in $\mathrm{HAC}$, in order to find efficient target therapies.

\section{References}

1. Qu BG, Bi WM, Qu BT, Qu T, Han XH, Wang H, Liu YX and Jia YG: PRISMA-Compliant Article: Clinical characteristics and factors influencing prognosis of patients with hepatoid adenocarcinoma of the stomach in China. Medicine (Baltimore) 95: e3399, 2016.

2. Ishikura H, Ishiguro T, Enatsu C, Fujii H, Kakuta Y, Kanda M and Yoshiki T: Hepatoid adenocarcinoma of the renal pelvis producing alpha-fetoprotein of hepatic type and bile pigment. Cancer 67: 3051-3056, 1991.

3. FornasaF: Soft-Tissue Localization of Hepatoid Adenocarcinoma: First case report. Case Rep Oncol 3: 212-217, 2010.

4. Yang J, Wang R, Zhang W, Zhuang W, Wang M and Tang C: Clinicopathological and prognostic characteristics of hepatoid adenocarcinoma of the stomach. Gastroenterol Res Pract 2014: 140587, 2014.

5. Nagai E, Ueyama T, Yao T and Tsuneyoshi M: Hepatoid adenocarcinoma of the stomach. A clinicopathologic and immunohistochemical analysis. Cancer 72: 1827-1835, 1993.

6. Xiao C, Wu F, Jiang H, Teng L, Song F, Wang Q and Yang H: Hepatoid adenocarcinoma of the stomach: Nine case reports and treatment outcomes. Oncol Lett 10: 1605-1609, 2015.

7. Liberati A, Altman DG, Tetzlaff J, Mulrow C, Gøtzsche PC, Ioannidis JP, Clarke M, Devereaux PJ, Kleijnen J and Moher D: The PRISMA statement for reporting systematic reviews and meta-analyses of studies that evaluate healthcare interventions: Explanation and elaboration. BMJ 339: b2700, 2009.

8. Baek SK, Han SW, Oh DY, Im SA, Kim TY and Bang YJ: Clinicopathologic characteristics and treatment outcomes of hepatoid adenocarcinoma of the stomach, a rare but unique subtype of gastric cancer. BMC Gastroenterol 11: 56, 2011.

9. Shimada S, Hayashi N, Marutsuka T, Baba Y, Yokoyama S, Iyama $\mathrm{K}$ and Ogawa M: Irinotecan plus low-dose cisplatin for alpha-fetoprotein-producing gastric carcinoma with multiple liver metastases: Report of two cases. Surg Today 32: 1075-1080, 2002.

10. Takeyama H, Sawai H, Wakasugi T, Takahashi H, Matsuo Y, Ochi N, Yasuda A, Sato M, Okada Y, Funahashi H, et al: Successful paclitaxel-based chemotherapy for an alpha-fetoprotein-producing gastric cancer patient with multiple liver metastases. World J Surg Oncol 5: 79, 2007.

11. Takahashi T, Kochi M, Kanamori N, Kaiga T, Funada T, Fujii M and Takayama T: Complete remission with FLEP chemotherapy for multiple liver metastasis from alpha-fetoprotein-producing gastric cancer-report of a case. Gan To Kagaku Ryoho 36 : 1885-1888, 2009 (In Japanese).
12. Gavrancic T and Park YH: A novel approach using sorafenib in alpha fetoprotein-producing hepatoid adenocarcinoma of the lung. J Natl Compr Cancer Netw 13: 387-391, 2015

13. Chang YC, Nagasue N, Kohno H, Ohiwa K, Yamanoi A and Nakamura T: Xenotransplantation of alpha-fetoprotein-producing gastric cancers into nude mice. Characteristics and responses to chemotherapy. Cancer 69: 872-877, 1992.

14. Metzgeroth G, Ströbel P, Baumbusch T, Reiter A and Hastka J: Hepatoid adenocarcinoma-review of the literature illustrated by a rare case originating in the peritoneal cavity. Onkologie 33: 263-269, 2010.

15. Chiba N, Yoshioka T, Sakayori M, Mikami Y, Miyazaki S, Akiyama S, Otsuka K, Yamaura G, Shibata H, Kato S, et al: AFP-producing hepatoid adenocarcinoma in association with Barrett's esophagus with multiple liver metastasis responding to paclitaxel/CDDP: A case report. Anticancer Res 25: 2965-2968, 2005.

16. Lin CW, Hsu CC, Chang HC, Sun YC, Sun PL, Hsu CY and Perng DS: Hepatoid adenocarcinoma of the stomach with liver metastasis mimicking hepatocellular carcinoma: A case report. Cases J 2: 6317, 2009.

17. Gálvez-Muñoz E, Gallego-Plazas J, Gonzalez-Orozco V, Menarguez-Pina F, Ruiz-Maciá JA and Morcillo MA: Hepatoid adenocarcinoma of the stomach-a different histology for not so different gastric adenocarcinoma: A case report. Int Semin Surg Oncol 6: 13, 2009.

18. Cappetta A, Bergamo F, Mescoli C, Lonardi S, Rugge M and Zagonel V: Hepatoid adenocarcinoma of the colon: What should we target? Pathol Oncol Res 18: 93-96, 2012.

19. Ye MF, Tao F, Liu F and Sun AJ: Hepatoid adenocarcinoma of the stomach: A report of three cases. World J Gastroenterol 19: 4437-4442, 2013.

20. Ahn JS, Jeon JR, Yoo HS, Park TK, Park CK, Sinn DH and Paik SW: Hepatoid adenocarcinoma of the stomach: An unusual case of elevated alpha-fetoprotein with prior treatment for hepatocellular carcinoma. Clin Mol Hepatol 19: 173-178, 2013.

21. Majumder S and Dasanu CA: Hepatoid variant of pancreatic cancer: Insights from a case and literature review. JOP 14: 442-445, 2013.

22. Nagai Y, Kato T, Harano M, Satoh D, Choda Y, Tokumoto N, Kanazawa T, Matsukawa H, Ojima Y, Idani $\mathrm{H}$, et al: A case of AFP-producing esophagogastric junction cancer with liver metastases with a good response to chemotherapy. Gan To Kagaku Ryoho 41: 2349-2351, 2014 (In Japanese).

23. Chen Y, Schaeffer DF and Yoshida EM: Hepatoid adenocarcinoma of the colon in a patient with inflammatory bowel disease. World J Gastroenterol 20: 12657-12661, 2014.

24. Hu CH, Li QL, Li HP, Fan SQ, Zhang HX, Liu XL, He Y, Huang M, Lu M, Wang SS and Wu F: Rare coexistence of mediastinal hepatoid adenocarcinoma, idiopathic azoospermia and horseshoe kidney: A case report and review of the literature. Int J Clin Exp Pathol 8: 11741-11746, 2015.

25. Karayiannakis AJ,Kakolyris S, Giatromanolaki A, Courcoutsakis N, Bolanaki H, Chelis L, Sivridis E and Simopoulos C: Hepatoid Adenocarcinoma of the Gallbladder: Case Report and Literature Review. J Gastrointest Cancer. 43: 139-144, 2012.

26. Petrelli F, Ghilardi M, Colombo S, Stringhi E, Barbara C, Cabiddu M, Elia S, Corti D and Barni S: A rare case of metastatic pancreatic hepatoid carcinoma treated with sorafenib. J Gastrointest Cancer 43: 97-102, 2012.

27. Mokrim M, Belbaraka R, Allaoui M, Kairaouani M, Mahassini N, Tahri A and Errihani H: Hepatoid Adenocarcinoma of the Lung: A Case Report and Literature Review. J Gastrointest Cancer. 43:125-127, 2012.

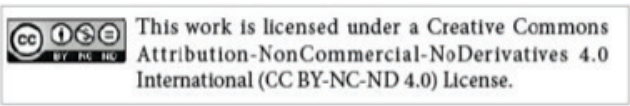

